\title{
Self-assembled ionophores based on 8-phenyl-2'-deoxyguanosine analogues
}

\author{
Vladimir Gubala, Doriann De Jesús and José M. Rivera* \\ Department of Chemistry, University of Puerto Rico, Río Piedras Campus, San Juan 00931, Puerto Rico
}

Received 24 October 2005; revised 15 December 2005; accepted 19 December 2005

\begin{abstract}
Novel base-modified G-analogues act as self-assembled ionophores for various metal cations. Their extracting efficiency can be modulated by the presence and substitution pattern of a functionalized phenyl group that replaces the $\mathrm{H} 8$ in the guanine base. (C) 2006 Published by Elsevier Ltd.
\end{abstract}

Selective and efficient ion complexation and transport across membranes by natural and synthetic ionophores are among the most widely studied phenomena in supramolecular chemistry. However, due to its many potential applications in environmental sciences and in sensor development, this subject is still of great relevance today, 38 years after Pedersen's seminal paper on the synthesis and characterization of crown ethers. ${ }^{1}$ Although there are numerous examples of synthetic ionophores that offer excellent selectivity for a variety of ions, one of the biggest challenges preventing their widespread use is the significant time and effort needed to synthesize them. To overcome this problem, the groups of Davis and Gottarelli have taken advantage of selfassembly to construct ionophores based on sugar-modified lipophilic guanosine analogues. These have been shown to have excellent properties in the extraction of alkaline and alkaline earth cations. ${ }^{2-8}$ Guanosine and related compounds have a propensity to form planar tetramers (G-tetrads), which are held together by $\mathrm{H}$-bonds and cation complexation (Fig. 1); further stacking of such tetrads leads to G-quadruplex formation. The potential of this strategy for cation complexation is illustrated by the exquisite and selective extraction, over a wide $\mathrm{pH}$ range, of the radioactive pollutant ${ }^{226} \mathrm{Ra}^{2+}$ by lipophilic G-quadruplexes even in the presence of excess alkali and alkaline earth cations. ${ }^{9}$

Recently we have demonstrated that the self-assembly of lipophilic dG-analogues could be modulated by replace-

\footnotetext{
Keywords: G-analogues; G-quadruplex; Self-assembly; Ionophores.

* Corresponding author. Tel.: +1 787 7640000x2906; fax: +1 787

7568242; e-mail: jrivera@cnnet.upr.edu
}

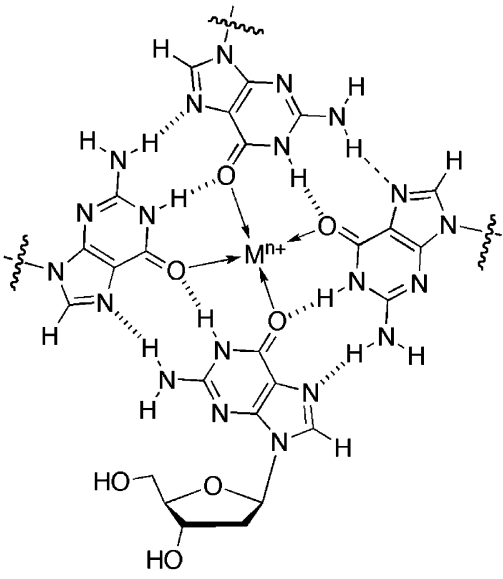

Figure 1. G-tetrad.

ment of the $\mathrm{H} 8$ in the guanine base with a functionalized phenyl group. ${ }^{10}$ In particular, the position of the functional group in the phenyl ring controlled the stoichiometry and thermal stability of the resulting supramolecules. Herein we report the first example of the use of base-modified dG-analogues to modulate the extraction selectivity toward a variety of metal cations. One of the most attractive features of this strategy is its potential for fine-tuning a desired selectivity, which could be readily engineered due to the ease of synthesis of a wide variety of 8-aryl-dG analogues.

Lipophilic G-analogues Gi, pAGi, and mAGi are available in high yields in three easy steps from $\mathbf{d G}$ and other readily available starting materials (Scheme 1). ${ }^{10,11}$ The compounds are soluble in chloroform in concentrations 


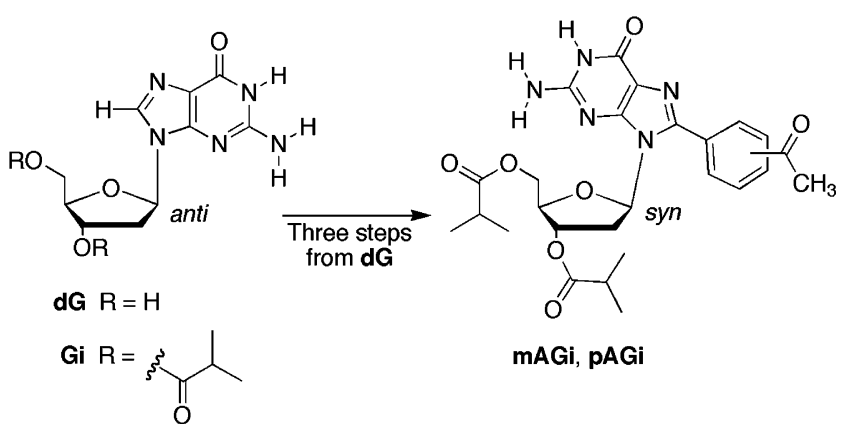

Scheme 1. Lipophilic G-analogues Gi, pAGi, and mAGi.

of up to $50 \mathrm{mM}$ and easily extract metal picrates from aqueous solutions. For this study, we evaluated the extraction efficiency of such compounds, defined as the ratio of ( $\mu \mathrm{mol}$ of metal extracted) $/(\mu \mathrm{mol}$ of analogue), at $5 \mathrm{mM}$ using an excess $(20 \mathrm{mM})$ of representative examples of alkaline $\left(\mathrm{Li}^{+}, \mathrm{Na}^{+}\right.$, and $\left.\mathrm{K}^{+}\right)$and alkaline earth $\left(\mathrm{Ba}^{2+}\right.$ and $\left.\mathrm{Sr}^{2+}\right)$ metal picrate salts. ${ }^{\dagger}$ The data shown in Figure 2 are for the extraction efficiency (light grey bars) grouped by analogues and by metals in order to facilitate the comparisons and evaluate any trends better. For example, parallel to the parent $\mathbf{G i}$, the analogue pAGi is more efficient at extracting alkaline cations than alkaline earth while $\mathbf{m A G i}$ shows no obvious preference between the two groups. In general, all analogues show only a small preference for $\mathrm{Sr}^{2+} \cdot \mathrm{Li}^{+}$ and $\mathrm{K}^{+}$are extracted relatively well by both the parent Gi and mAGi but pAGi is extremely efficient at extracting the same cations.

The fact that these compounds extracted (extraction efficiency $\geqslant 0.125$ ) more than one cation per tetrad suggests that there might be other binding sites in addition to the quadruplex internal cavity. To assess this and establish the differences in binding affinities between the different binding sites, we performed back extraction experiments (darker bars on Fig. 2). Notice that both Gi and mAGi drop their metal cation content to about one per octamer with the former releasing almost all of the lithium picrate. In sharp contrast, pAGi maintains extraction efficiencies greater than 0.45 , which indicates that its octamer remains bound to an average of four cations of lithium and potassium!

Although, we expected to see significant differences between the parent $\mathbf{G i}$ and the two analogues, the differ-

\footnotetext{
${ }^{\dagger}$ Picrate Extraction Conditions: A solution of the analogue in $\mathrm{CHCl}_{3}$ $(2 \mathrm{~mL}, 5 \mathrm{mM})$ was mixed with a solution of aqueous picrate $(2 \mathrm{~mL}$, $20 \mathrm{mM})$. After stirring $(1 \mathrm{~h})$, centrifuging $(10 \mathrm{~min})$ the mixture, the aqueous phase was separated and its absorbance at $355 \mathrm{~nm}$ was measured in a UV-vis spectrometer (Cary 50). The back extraction experiments were performed by taking $1 \mathrm{~mL}$ of the organic phase and stirring $(1 \mathrm{~h})$ it with $1 \mathrm{~mL}$ of nanopure water, centrifuging $(10 \mathrm{~min})$ and measuring the aqueous phase to account for the released metal picrate. For both the extraction and back extraction experiments, the amount of metal picrate was determined using a calibration curve applying the Beer-Lambert's law and the values are the average of four measurements.
}

ences between mAGi and pAGi are particularly striking when one considers that the 'only' change between these two analogues is the position of the acetyl group attached to the phenyl ring (para vs meta). The fact that pAGi shows the highest extraction efficiency of the three compounds and that it is able to hold on to the metal picrates even after back extraction prompted us to perform molecular modeling studies in order to explain such phenomena. ${ }^{*}$ The octamer $(\mathbf{p A G i})_{8}$ can hold up to six lithium cations as illustrated in Figure 3 (similar studies show that up to five potassium and sodium cations could be accommodated as well). The paraacetyl groups in all the pAGi monomers converge with the carbonyl of the $5^{\prime}$-isobutyric ester groups to form a nice pocket of an appropriate size that fits $\mathrm{Li}^{+}$, $\mathrm{Na}^{+}$, and $\mathrm{K}^{+}$. Hydrophilic G-quadruplexes made by 5'-GMP show an analogous binding behavior for surface complexation of cations in addition to complexation in the internal cavity. ${ }^{12}$

After extraction experiments the content of the organic layer was evaluated by ${ }^{1} \mathrm{H}$ NMR in order to corroborate the UV-vis studies and to gain a better understanding of the type of quadruplexes formed (Fig. 4). The partial spectra for mAGi illustrates the formation of octamers $(\mathrm{O})$ with all the metal cations except for potassium, which also templated the formation of a hexadecamer $(\mathrm{H})$. The picrate anion peak also changes its chemical shift (up to $0.2 \mathrm{ppm}$ ) indicating different levels of interaction with the complexes. The peaks for the complexes with potassium and sodium cations are significantly sharper than those with the other cations, which suggest that the former are kinetically more stable.

Similar to covalent ionophores, the extracting selectivity of self-assembled ionophores is influenced by a large number of factors some of which are (i) cavity size; (ii) charge density; (iii) solvent; (iv) preorganization of host; (v) solvation energy; (vi) binding kinetics; (vii) the nature of the counter-anion and its interactions with solvent, cation, and complex. ${ }^{13}$ The latter has been documented for the picrate anion, which can affect cation complexation due to the high diversity of both the coordination and geometry of the picrate-cation interactions. ${ }^{14}$ For example, the intensity of picrate interactions with supramolecular complexes has been shown to be crucial for the stabilization of the lipophilic G-quadruplexes studied by the Davis' group. ${ }^{4} \mathrm{X}$-ray and NMR evidence of strong and well-defined interactions between the picrate anions and available hydrogen bonding sites in the grooves of the quadruplexes were shown to be essential for complex formation. The related compound $\mathbf{G i}$ is expected to benefit from such strong interactions with the picrate anions in contrast to $\mathbf{m A G i}$ and $\mathbf{p A G i}$ in which the phenyl group should minimize such interactions making their extracting efficiency relatively anion independent.

\footnotetext{
Molecular modeling was performed using HyperChem 7.5 for Windows (from Hypercube, Inc.) with the Amber 99 force field.
} 


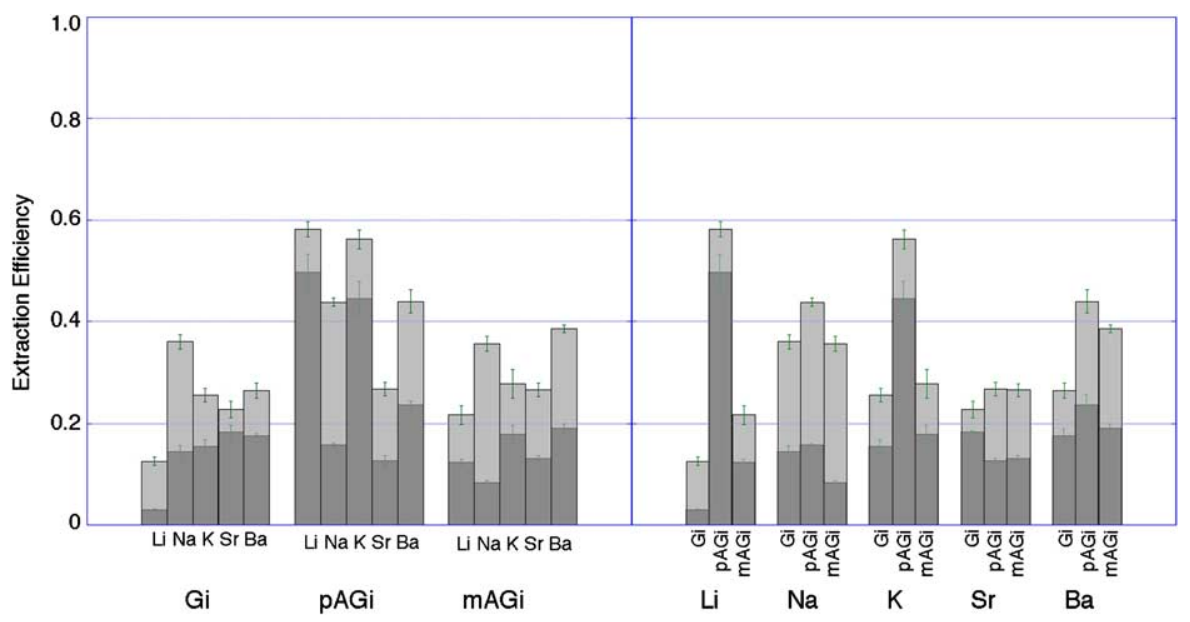

Figure 2. Extraction efficiency ( $\mu \mathrm{mol}$ cation/ $\mu \mathrm{mol}$ analogue) profiles grouped by analogues (left) and by the type of metals (right) as determined by UV-vis experiments. The light grey bars represent the extraction experiments and the dark grey bars represent the amount of metal picrate left after back extraction. The experiments were performed with chloroform as the organic phase and the results are the average value of four measurements. ${ }^{\dagger}$
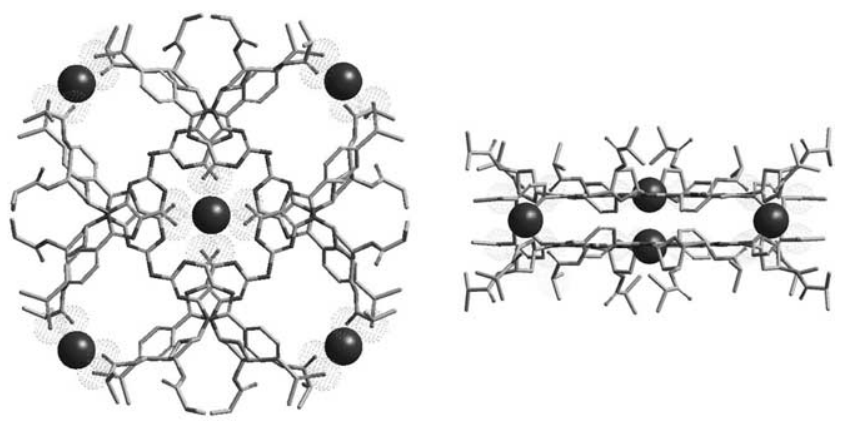

Figure 3. Top (left) and side (right) views of a computer generated molecular model of the proposed pAGi octamer with up to six $\mathrm{Li}^{+}$ cations bound and represented as black spheres.

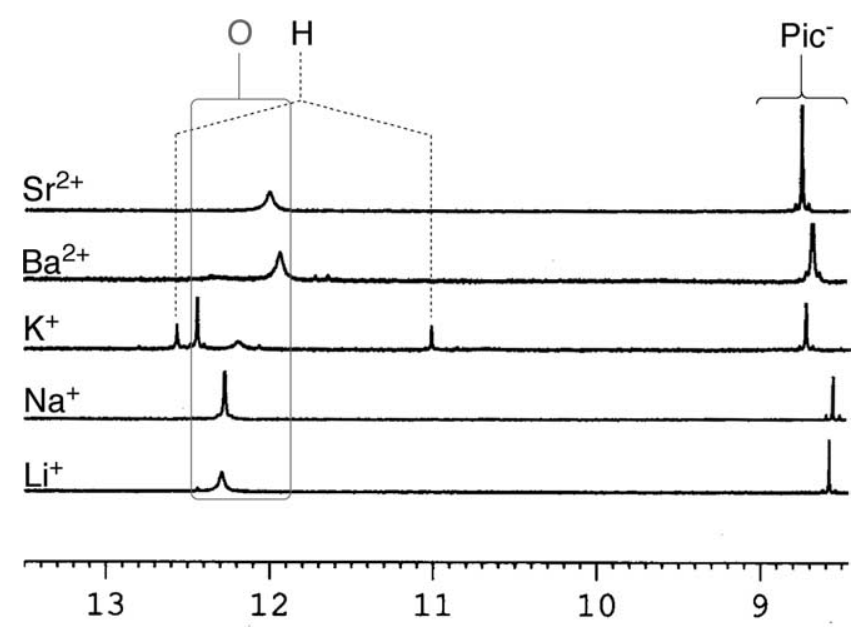

Figure 4. Partial ${ }^{1} \mathrm{H}$ NMR spectra in $\mathrm{CDCl}_{3}(295 \mathrm{~K}, 500 \mathrm{MHz})$ showing the region of the $\mathrm{N}^{1} \mathrm{H}(11-13 \mathrm{ppm})$ and the peak of the picrate anion $(\sim 8.5 \mathrm{ppm})$ obtained from the organic layer after extraction of $\mathrm{M}(\mathrm{Pic})_{n}(20 \mathrm{mM})$ with $\mathbf{m A G i}(5 \mathrm{mM})$.

Another factor that is likely influencing the extracting efficiency of these compounds is related to their propen- sity to form quadruplexes of different stoichiometries. The presence of the 8-phenyl group preorganizes the structures of $\mathbf{m A G i}$ and $\mathbf{p A G i}$ in the syn conformation (Scheme 1), which avoids the formation of the columnar aggregates (CA) produced by $\mathbf{G i}$, stopping the assembly at either an octameric $(\mathrm{O})$ or a hexadecameric $(\mathrm{H})$ structure (Fig. 5d). Furthermore, the extended Hoogsteen edge of mAGi enhances the number of hydrogen bonds formed between the monomers in the tetrads relative to both Gi and pAGi (Fig. 5a-c). ${ }^{10}$
a. $(\mathbf{G i})_{4}$
b. $(\mathbf{p A G i})_{4}$
c. $(\mathbf{m A G i})_{4}$
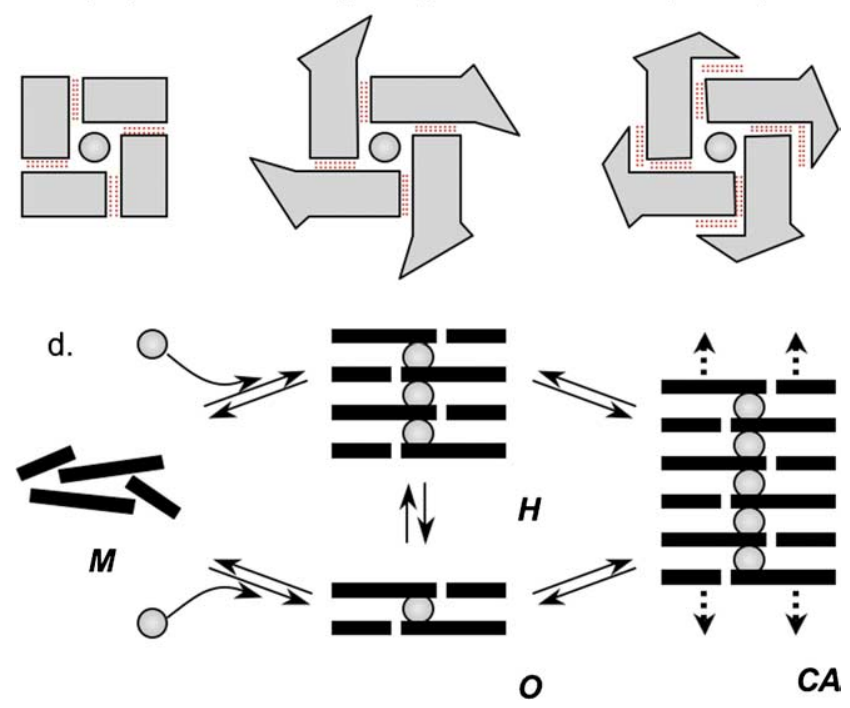

Figure 5. (a-c, top view): Cartoon representations of the tetrads formed by the lipophilic G-analogues Gi, pAGi, and mAGi. The dotted lines between the subunits represent the hydrogen bonds made by such structures. The gray circle represents a cation $\mathrm{M}^{n+}$. (d, side view): Schematic representation of the hierarchical self-assembly of the lipophilic G-analogues with concomitant complexation of a metal cation $\mathrm{M}^{n+}$ (gray circle). ( $\boldsymbol{M}=$ loosely organized Monomers, $\boldsymbol{O}=O$ ctamer, $\boldsymbol{H}=$ Hexadecamer and $\boldsymbol{C A}=$ Columnar $A$ ggregates $)$. 
In summary, the differences in the extracting profiles shown here underscore the key finding of this study, which is that seemingly simple substitutions on the guanine base can alter dramatically the selectivity of the resulting quadruplex for a given cation. Therefore this offers a convenient avenue for the development of selective self-assembled ionophores from base-modified analogues. In addition to the enhanced selectivity, we have also identified a strategy to use the functional groups on the periphery of these supramolecules to increase the binding capacity by creating extra binding cavities. Currently available with us is a library of more than fifty different base- and sugar-modified lipophilic G-analogues that are being evaluated as self-assembling ionophores with potentially enhanced affinities and selectivities. The results of extraction studies with such compounds as well as thermodynamic and kinetic characterization experiments will be presented in the near future.

\section{Acknowledgements}

We thank the NIH-SCoRE program for financial support, the National Science Foundation EPSCoR program for a graduate fellowship to V.G. and the NIH-MARC program for an undergraduate fellowship to D.D.J.

\section{References and notes}

1. Pedersen, C. J. J. Am. Chem. Soc. 1967, 89, 7017-7036.

2. Spada, G. P.; Gottarelli, G. Synlett 2004, 596-602.

3. Davis, J. T. Angew. Chem., Int. Ed. 2004, 43, 668-698.

4. Shi, X. D.; Mullaugh, K. M.; Fettinger, J. C.; Jiang, Y.; Hofstadler, S. A.; Davis, J. T. J. Am. Chem. Soc. 2003, 125, 10830-10841.

5. Mezzina, E.; Mariani, P.; Itri, R.; Masiero, S.; Pieraccini, S.; Spada, G. P.; Spinozzi, F.; Davis, J. T.; Gottarelli, G. Chem. Eur. J. 2001, 7, 388-395.

6. Tirumala, S.; Davis, J. T. J. Am. Chem. Soc. 1997, 119, 2769-2776.

7. Gottarelli, G.; Masiero, S.; Spada, G. P. J. Chem. Soc., Chem. Commun. 1995, 2555-2557.

8. Davis, J. T.; Tirumala, S.; Jenssen, J. R.; Radler, E.; Fabris, D. J. Org. Chem. 1995, 60, 4167-4176.

9. van Leeuwen, F. W. B.; Verboom, W.; Shi, X. D.; Davis, J. T.; Reinhoudt, D. N. J. Am. Chem. Soc. 2004, 126, 16575-16581.

10. Gubala, V.; Betancourt, J. E.; Rivera, J. M. Org. Lett. 2004, 6, 4735-4738.

11. Western, E. C.; Daft, J. R.; Johnson, E. M.; Gannett, P. M.; Shaughnessy, K. H. J. Org. Chem. 2003, 68, 67676774.

12. Wong, A.; Wu, G. J. Am. Chem. Soc. 2003, 125, 1389513905.

13. Steed, J. W.; Atwood, J. L. Supramolecular Chemistry; John Wiley \& Sons: New York, 2000.

14. Olsher, U.; Feinberg, H.; Frolow, F.; Shoham, G. Pure Appl. Chem. 1996, 68, 1195-1199. 